\title{
Influence of cholesterol on the structure of stratum corneum lipid model membrane
}

\author{
J. Zbytovskáa,b,*, M.A. Kiselev ${ }^{\text {b,c }}$, S.S. Funari ${ }^{\mathrm{d}}$, V.M. Garamus ${ }^{\mathrm{e}}$, S. Wartewig ${ }^{\mathrm{f}}$, K. Palát $^{\mathrm{g}}$, R. Neubert ${ }^{\mathrm{b}}$ \\ a Department of Pharmaceutical Technology, Faculty of Pharmacy, Charles University in Prague, Heyrovského 1203, 50005 Hradec Králové, Czech Republic \\ ${ }^{\mathrm{b}}$ Department of Pharmacy, Martin-Luther-University Halle-Wittenberg, Wolfgang-Langenbeck Str. 4, D-06120 Halle/Saale, Germany \\ c Frank Laboratory of Neutron Physics, Joint Institute for Nuclear Research, Dubna 141980, Russia \\ ' HASYLAB, Notkestrasse 85, D-22607 Hamburg, Germany \\ e GKSS Research Centre, Max-Planck-Str., D-21502 Geesthacht, Germany \\ ${ }^{\mathrm{f}}$ Institute of Applied Dermatopharmacy, Wolfgang-Langenbeck Str. 4, D-06120 Halle/Saale, Germany \\ g Department of Inorganic and Organic Chemistry, Faculty of Pharmacy, Charles University in Prague, Heyrovského 1203, 50005 Hradec Králové, Czech Republic
}

\section{A R T I C L E I N F O}

\section{Article history:}

Received 16 September 2007

Received in revised form 2 June 2008

Accepted 17 June 2008

Available online 22 June 2008

\section{Keywords:}

Stratum corneum lipids

Cholesterol

Recessive X-linked ichthyosis

X-ray diffraction

Neutron scattering

Unilamellar vesicles

\begin{abstract}
A B S T R A C T
This study describes the influence of cholesterol on a model membrane consisting of four stratum corneum (SC) lipids in three different states at 32 and $85^{\circ} \mathrm{C}$. Using small-angle X-ray diffraction on multilamellar vesicles, the lamellar repeat distance, $D$, was determined. Small-angle neutron scattering on unilamellar vesicles was used to calculate the membrane thickness, average area of the membrane surface per molecule, average volume per molecule in the membrane, and membrane density. After the sample preparation, the membranes show one lamellar phase. Over 10 days, the systems separate into two lamellar phases and crystalline cholesterol. On heating the samples to $85^{\circ} \mathrm{C}$, the domains merge into one phase, the $D$-value of which increases slightly with increasing cholesterol concentration. On cooling the samples back to $32^{\circ} \mathrm{C}$, only one phase is observable again. The increasing cholesterol concentration in the membrane causes a decrease in the $D$-value, membrane thickness, and membrane density. Simultaneously, the area of the membrane surface and membrane volume per molecule increase. In conclusion, cholesterol fluidises the SC lipid membranes in the state below the main phase transition and condenses above it. This effect can have an important impact on the pathologic skin states such as the recessive X-linked ichthyosis.
\end{abstract}

(C) 2008 Elsevier B.V. All rights reserved.

\section{Introduction}

The skin barrier plays an essential role in protecting the body against xenobiotics from the environment and against water evaporation from the organism. Stratum corneum (SC), the outermost skin layer, is responsible for the permeability of mammalian skin. This membrane consists of keratin-rich corneocytes embedded in a lipid matrix with a lamellar organization. Ceramides of nine types, free fatty acids, cholesterol, and cholesterol derivatives are the most abundant lipids in the SC [1].

The SC lipids show a complex behaviour, which is dependent on the particular lipid composition in the SC matrix. In diseased skin, a deviation in lipid composition has often been

\footnotetext{
* Corresponding author at: Faculty of Pharmacy, Charles University in Prague, Department of Pharmaceutical Technology, Heyrovského 1203, 50005 Hradec Králové, Czech Republic. Tel.: +420 495067383; fax: +420 495518002.

E-mail address: zbytovska@faf.cuni.cz (J. Zbytovská).
}

found. The recessive X-linked ichthyosis (RXLI) is associated with the steroid sulphatase deficiency; the enzyme which converts sulphated steroids to steroids [2]. Therefore, increased levels of cholesterol sulphate (CS), connected with decreased levels of cholesterol (CHOL), were found in the affected skin [3]. Although the CS accumulation seems to be the primary mechanism contributing to the barrier abnormalities in RXLI, the reduced $\mathrm{CHOL}$ levels also play an important role, especially in the altered membrane dynamics. Topically administrated CHOL reverses the pathologic effects of excess CS on SC membrane structure, barrier function, and desquamation [4]. Additionally, ichthyotic symptoms can be induced by cholesterol-lowering drugs [5].

Although there are several studies focusing on the role of $\mathrm{CHOL}$ in the SC [6-9], the influence of CHOL on the SC lipid membrane structure still requires a complete elucidation.

There is a range of methods, such as X-ray diffraction, NMR, IR and Raman spectroscopy that can be used to describe the internal structure of membranes. Small-angle neutron scattering (SANS) is 


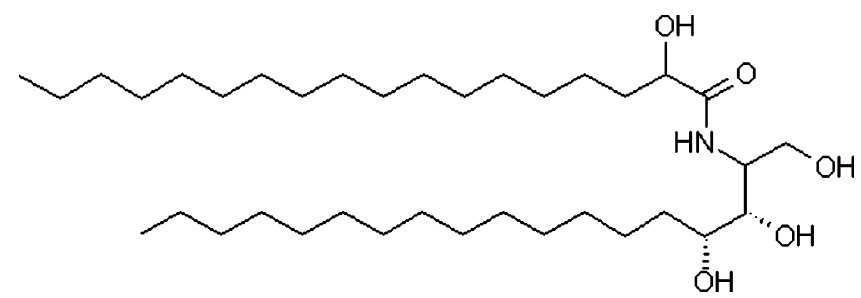

Fig. 1. Chemical structure of Cer[AP].

not an unusual technique in the studies on various phospholipid systems [10,11]; it has, however, rarely been employed in the skin characterization. The very small-angle neutron scattering has been successfully applied to study the water sorption [12] and to calculate some structural features of SC [13]. Similarly, the neutron diffraction on native SC $[14,15]$ and multilamellar SC lipid films [16] makes it possible to study the SC membrane structure and hydration. Nevertheless, SANS has been reported not to be the ideal method when used on the native SC due to the high complexity of the system [17].

For this reason, SANS on a more simple system such as unilamellar vesicles (ULVs) of a SC lipid model membrane is employed in this study. Because it is not possible to prepare stable ULVs at a pH close to the physiological pH of human skin [18], the experiments must be carried out at higher $\mathrm{pH}$ values when the state of protonation of the charged membrane components changes.

The aim of the present study is to describe the effect of $\mathrm{CHOL}$ on a model membrane imitating the native SC lipid matrix and to characterize the alterations in the membrane structure evoked by a change of $\mathrm{CHOL}$ concentration in the system. Besides X-ray diffraction on multilamellar vesicles (MLVs), SANS on ULVs is newly applied. The obtained results should contribute to elucidation of CHOL in RXLI.

\section{Material and methods}

\subsection{Material}

$N$ - $\alpha$-Hydroxyoctadecanoylphytosphingosine (Cer[AP]; see Fig. 1) was a gift from Cosmoferm (Delft, The Netherlands). CHOL CS, and palmitic acid, as well as Trizma ${ }^{\circledR}$ (Tris) buffer, and sodium chloride were purchased from Sigma-Aldrich (Taufkirchen, Germany). $\mathrm{D}_{2} \mathrm{O}$ (99.98\% deuteration) was purchased from Chemotrade (Leipzig, Germany). Water, chloroform, and methanol used were of HPLC grade.

\subsection{Vesicle preparation}

The composition of the lipid system used in this study was chosen based on previous data $[18,19]$. A basic system (mixture IV) consisting of $55 \%$ (in weight) Cer[AP], 25\% CHOL, 15\% palmitic acid, and $5 \%$ CS should mostly imitate the real SC lipid composition. The other samples were prepared by varying the proportion of $\mathrm{CHOL}$ (from 10 to $30 \%$ ), with the relative ratio of other lipids remaining constant. The samples used in the study are listed in Table 1.

MLVs were prepared by the 'thin layer method' [20]. The lipids were dissolved separately in a chloroform/methanol mixture 2:1 (in volume). The required amounts of the solutions were mixed together and dried using a rotary evaporator. To remove completely the solvent, the samples were kept under vacuum for 1 day. An appropriate amount of $10 \mathrm{mM}$ Tris buffer, pH 9.0 with $100 \mathrm{mM} \mathrm{NaCl}$ in water or in $\mathrm{D}_{2} \mathrm{O}$ was added to the dry sample. The samples were then heated for $1 \mathrm{~h}$ to $90^{\circ} \mathrm{C}$ and mixed on a vortex every 20 min until a milky MLV suspension was formed.
The ULVs were prepared from the MLV suspension by extrusion through polycarbonate filters with a pore diameter of $500 \AA$ at $75^{\circ} \mathrm{C}$ using a LiposoFast Basic extruder from Avestin (Ottawa, Canada).

\subsection{Vesicle characterization}

The concentration of lipids in the ULVs after extrusion was determined by high performance thin layer chromatography (HPTLC) using Automatic TLC Sampler 4, AMD 2 development chamber and TLC scanner 3 (Camag, Muttenz, Switzerland) according to Farwanah et al. [21]. Integration and quantification based on peak areas were performed using CATS software (Camag).

The size and stability of prepared ULVs $(1 \%, \mathrm{w} / \mathrm{w}$ of lipids in buffer) was checked by photon correlation spectroscopy using a particle size analyser (Malvern HPPS-ET, Malvern Instruments, UK). The measurements were carried out at $32^{\circ} \mathrm{C}$. The hydrodynamic radius and the polydispersity have been calculated from the correlation function by the CONTIN algorithm using the HPPSMalvern program for dispersion technology and light scattering systems.

\subsection{Small-angle X-ray diffraction}

Small-angle X-ray diffraction (SAXD) data were collected on the Soft Condensed Matter beamline A2 of HASYLAB at the storage ring Doris III of the Deutsches Elektronen Synchrotron. A two-dimensional CCD detector was used for data acquisition. The diffraction patterns from MLVs with $20 \%(\mathrm{w} / \mathrm{w}$ ) lipid concentration in Tris buffer with $100 \mathrm{mM}$ sodium chloride were measured at 32,85 , and again at $32^{\circ} \mathrm{C}$ in specially designed copper cells $(50 \mu \mathrm{l}$ in volume) with a polyimid-foil (Kapton ${ }^{\circledR}$, DuPont, Luxembourg) window. Before the measurements, the samples were incubated 10 days at $5{ }^{\circ} \mathrm{C}$. The sample-to-detector distance was $585 \mathrm{~mm}$ and the $\mathrm{X}$-ray wavelength was $1.5 \AA$. The acquisition time of each sample was $3 \mathrm{~min}$. Silver behenate and rat tendon tail were used for calibration. Prior to each measurement, the sample was allowed to thermally equilibrate for $10 \mathrm{~min}$.

The data evaluation was carried out using the FIT2D software [22]. The scattered intensity was measured as a function of scattering vector, $q$. The latter is defined as $q=(4 \pi / \lambda) \sin \theta$, where $2 \theta$ is the scattering angle and $\lambda$ is the X-ray wavelength. The lamellar repeat distance, $D$, was calculated from the first order diffraction peak according to $D=2 \pi / q$. The diffraction peaks were fit using a Lorentzian function to determine the exact positions. This function was chosen instead of the usual Gaussian one because of the higher fit accuracy.

A STOE STADI-IV diffractometer (Stoe and Cie, Darmstadt, Germany), equipped with $\mathrm{Cu} K \alpha$ radiation (wavelength $1.54 \AA$ ) and a linear position sensitive detector, was used to measure the diffraction patterns from the MLVs about $3 \mathrm{~h}$ after the preparation. The samples were placed in a quartz capillary and measured in a transmission geometry at $20^{\circ} \mathrm{C}$ with the $2 \theta$ scan between $0^{\circ}$ and $6^{\circ}$ taking $2 \mathrm{~h}$.

Table 1

Lipid composition of the model systems (in wt\%)

\begin{tabular}{llllll}
\hline Lipid & I & II & III & IV & V \\
\hline Cholesterol & $10(8.6)$ & $15(14.2)$ & $20(-)^{\mathrm{a}}$ & $25(21.6)$ & $30(27)$ \\
Ceramide[AP] & 66 & 62.3 & 58.7 & 55 & 51.3 \\
Palmitic acid & 18 & 17 & 16 & 15 & 14 \\
Cholesterol sulphate & 6 & 5.7 & 5.3 & 5 & 4.7
\end{tabular}

The concentration of cholesterol in ULVs after extrusion is given in brackets

a Not measured. 


\subsection{Small-angle neutron scattering}

The ULVs with $1 \%(\mathrm{w} / \mathrm{w})$ lipid concentration in Tris buffer in $\mathrm{D}_{2} \mathrm{O}$ adjusted to $\mathrm{pH} 9$ were measured with neutrons of wavelength $8.1 \AA$ at the SANS 1 spectrometer of the Geesthacht Neutron Facility, GKSS Research Centre, Germany. To obtain scattering curves in a broad q range, four sample-to-detector distances of 705.0, 1805.0, 4505.0, and $9705.0 \mathrm{~mm}$ were used. The data were collected at $32^{\circ} \mathrm{C}$. The acquisition time at $705.0 \mathrm{~mm}$ was $1 \mathrm{~h}$; at other sample-to-detector distances it was $0.5 \mathrm{~h}$. For background subtraction, the scattering curve of the relevant buffer has been used, which was measured the same way as the sample.

The analysis of the SANS curves has been performed using two methods, namely the 'model of separated form factors' (MSFF) [23], and the Kratky-Porod analysis of the Guinier approximation (GA) [24-26].

According to the MSFF, the macroscopic cross-section of the monodispersed population of ULVs is given by

$\frac{\mathrm{d} \Sigma(q)}{\mathrm{d} \Omega_{\mathrm{mon}}}=n F_{\mathrm{s}}(q, R) F_{\mathrm{b}}(q, d) S(q)$

where $n$ is the number of vesicles per unit volume, $F_{\mathrm{s}}(q, R)$ is the form factor of the infinitely thin sphere with the radius $R$

$F_{\mathrm{S}}(q, R)=\left(4 \pi \frac{R^{2}}{q R} \sin (q R)\right)^{2}$

$F_{\mathrm{b}}(q, d)$ is the form factor of the symmetrical lipid bilayer with the thickness $d$, which can be expressed by

$F_{\mathrm{b}}(q, d)=\left(\frac{2 \Delta \rho}{q} \sin \left(\frac{q d}{2}\right)\right)^{2}$

for the case of a bilayer with constant scattering-length density across the membrane, $\rho(x)=$ const. $\Delta \rho$ is the neutron contrast. $S(q)$ is structure factor of the vesicle population. For the used lipid concentrations $1 \%(\mathrm{w} / \mathrm{w})$ this factor is $S(q) \approx 1$ [27].

The average vesicle radius $R$ can be calculated from the scattering curve based on Eq. (2) as $R=\pi / q_{\mathrm{R} \min }$, where $q_{\mathrm{R} \text { min }}$ is the first minimum in the form factor of infinitely thin spheres after averaging the population of polydisperse vesicles [23,28].

The membrane thickness parameter $d$ can be directly calculated from the position $q_{0}$ of the first minimum of the sine function in Eq. (3) as $q=2 \pi / q_{\mathrm{d} \min }$. For a membrane thickness of about $30 \AA$, the position of $q_{\mathrm{d} \text { min }}$ is about $0.2 \AA^{-1}$ [28].

Another possibility to calculate the membrane thickness from a scattering curve is given by the GA [24-26]. In the $q$ range valid for a homogeneous membrane approximation, the scattering intensity of ULVs dispersed in heavy water can be given by

$I(q)=2 \pi I(0) q^{-2} \exp \left(-q^{2} R_{\mathrm{t}}^{2}\right)$

where $I(0)$ is scattering to 'zero angle' and $R_{\mathrm{t}}$ is membrane gyration radius. In this approach, the $R_{\mathrm{t}}$ parameter is the absolute value of the slope of the Kratky-Porod plot $\left(\ln \left[I(q) q^{2}\right]\right.$ versus $\left.q^{2}\right)$ and the membrane thickness parameter, $d_{\mathrm{g}}$, can be calculated as

$d_{\mathrm{g}}^{2}=12 R_{\mathrm{t}}^{2}$

$I(0)$ cannot be measured experimentally, but it can be determined by extrapolation of the Kratky-Porod plot to zero. The value of $I(0)$ is given by the total particle scattering-length, namely, by the sum of the scattering-lengths of all atoms inside the particle. Therefore, the chemical composition being known, the evaluation of $I(0)$ allows the molecular mass per unit of vesicle surface to be determined $[25,29]$. In the limit of $q \rightarrow 0$, the mass of the membrane per unit of surface, $M_{\mathrm{s}}$, can be determined by dividing the scattered intensity $I(0)$ by the total lipid concentration $\mathrm{c}$ and the average scattering-length density per unit mass, $\Delta \rho_{\mathrm{m}}$ according to

$I(0)=M_{\mathrm{s}} c \Delta \rho_{\mathrm{m}}^{2}$

The membrane area per molecule, $A$, in centrosymmetric bilayers can be calculated by

$A=\frac{2}{M_{\mathrm{S}} N_{\mathrm{A}} / M_{\mathrm{W}}}$

where $M_{\mathrm{W}}$ is the average molecular weight of the lipids, $M_{\mathrm{S}}$ the determined membrane mass per unit of surface and $N_{\mathrm{A}}$ the Avogadro number. In the present study, a four-component system is investigated. As it is not possible to determine the area per molecule for each component in the system, a term 'average area per molecule' is introduced.

Average volume per molecule in the membrane, $V$, has been calculated by multiplying the average area per molecule by half of the membrane thickness parameter [30]. Consequently, the average membrane density, $\rho_{\mathrm{m}}$, in $\mathrm{g} / \mathrm{cm}^{3}$ has been determined by dividing $V$ by the average weight of one molecule in the membrane, $M_{\mathrm{W}} \cdot M_{\mathrm{W}}$ has been calculated separately for every $\mathrm{CHOL}$ concentration and the weight ratio of the individual components was remained the same.

\subsection{Molecular modelling}

In order to determine the average excess scattering-length density per unit mass, the molecular volumes were calculated. Quantum-chemical calculations were run on a PC computer using software HyperChem for Windows v. 7.1, Hypercube Inc. The models of compounds were formed on RHF/AM1 level. We used the conformation of Cer[AP] with parallel aliphatic chains calculated as described previously [31]. Solvent accessible volumes, $V_{\mathrm{SA}}$, of models of studied compounds were calculated by the grid method [32] using the atomic radii of Gavezzotti [33]. The solvent probe radius $0.4 \AA$ and 20 points on the cube side were used.

\section{Results}

\subsection{Small-angle X-ray diffraction from MLVs}

Fig. 2 shows the diffraction patterns of the SC lipid model MLVs with 10 and 25\% CHOL (mixtures I and IV, respectively) measured

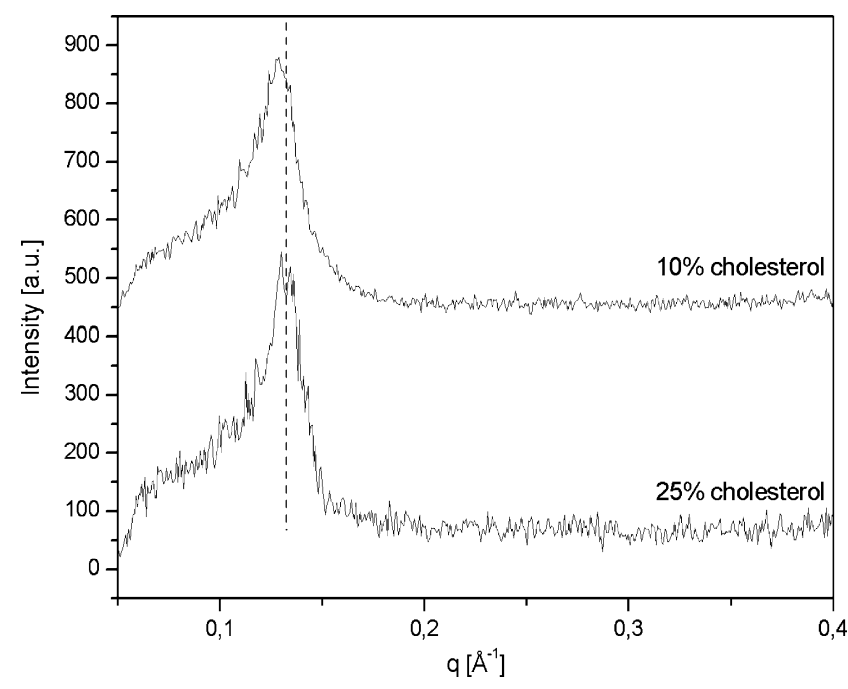

Fig. 2. X-ray diffraction patterns from the systems with 25 and $10 \%$ CHOL measured $3 \mathrm{~h}$ after the sample preparation. 
immediately after the preparation. The samples show only one diffraction peak at $0.137 \AA$ for $25 \% \mathrm{CHOL}$ and 0.131 Å for $10 \% \mathrm{CHOL}$, which indicates one phase with the lamellar repeat distance of 45.9 and $48.0 \AA$ A, respectively.

The diffraction patterns of the SC lipid model systems measured by synchrotron radiation, 10 days after the sample preparation with various $\mathrm{CHOL}$ concentrations are given in Fig. 3a-c. The obtained results are summarized in Table 2.

At $32^{\circ} \mathrm{C}$ before heating, the diffractograms are quite complex (Fig. 3a). For the purpose of clarity, we have called this state 'state $\alpha$ '. The positions of the diffraction peaks indicate that the systems are separated into two lamellar phases, namely a 'short' one (the $S$ phase) with the lamellar repeat distance of about $42 \AA$ and a 'long' one (the L-phase) with the lamellar repeat distance of about $47 \AA$. Both phases induce three diffraction orders in the diffractograms. It is apparent in Fig. 4 that with increasing CHOL content from 10 to $30 \%$ the calculated periodicity of the L-phase increases slightly from $46.7 \pm 0.2$ to $47.5 \pm 0.2 \AA$, whereas the periodicity of the S-phase remains almost unchanged.

In all the samples, a small peak is detectable at $0.187 \AA^{-1}$. This peak position corresponds to a repeat distance of $33.6 \pm 0.1 \AA$ and was assigned to crystalline CHOL monohydrate [34]. The peak intensity increases with the increasing concentration of CHOL in the mixture, while the position is constant. In the diffractograms of the samples with 25 and 30\% CHOL, even more diffraction orders of crystalline $\mathrm{CHOL}$ monohydrate are detectable.

When the systems are heated to $85^{\circ} \mathrm{C}$ (state $\beta$ ), the S- and Lphases merge into one phase, the repeat distance of which increases with the CHOL content from $41.8 \pm 0.2 \AA$ for the sample with $10 \%$ CHOL to $42.8 \pm 0.2 \AA$ for the sample with $30 \%$ CHOL (Figs. 3b and 4). Considering the peak at $0.187 \AA^{-1}$, a small amount of crystalline CHOL is still present, particularly in the samples with higher CHOL concentrations.

When the samples are cooled back to $32^{\circ} \mathrm{C}$, they show only one lamellar phase (state $\delta$ ). This phase is strongly affected by the concentration of $\mathrm{CHOL}$ in the system. The periodicity decreases almost linearly with increasing CHOL concentration from $47.6 \pm 0.2 \AA$ for $10 \%$ CHOL to $44.9 \pm 0.2 \AA$ for $30 \%$ CHOL (Figs. $3 \mathrm{c}$ and 4 ). The peak belonging to crystalline $\mathrm{CHOL}$ is weak yet observable, especially in the samples with higher CHOL content.

The recovery of the system with $25 \%$ CHOL (mixture IV) into the initial state $\alpha$ has been studied further. Fig. 5 shows the Xray diffraction patterns of the sample measured $20 \mathrm{~min}, 1$ day, and 10 days after the heating. Twenty minutes after the heating, there is one diffraction peak at $0.138 \AA^{-1}$, which indicates one phase with $45.5 \AA$. One day later, the sample still shows only one peak at $0.136 \AA^{-1}$ which corresponds to the $D$-value of $46.2 \AA$. The diffractogram measured 10 days after the heating shows two peaks indicating the phase separation into the $\mathrm{S}$ - and L-phases with 41.9 and $47.3 \AA$, respectively, and the peak of crystalline CHOL at $33.6 \AA$.

\subsection{Characterization of ULVS}

The HPTLC analysis of the lipids in the ULV and MLV samples confirms that the substances are stable during the sample preparation and shows that the $\mathrm{CHOL}$ content was slightly reduced after the extrusion. The concentration of CHOL in the ULVs is given in brackets in Table 1 . The difference in the proportions of the other lipids in MLVs and ULVs was negligible.

According to DLS studies, all the ULV samples showed a monomodal population with a hydrodynamic radius between 550 and $650 \AA$ and a polydispersity of about 30\%. Fig. 6 shows the size distribution of the sample with $25 \%$ CHOL (mixture
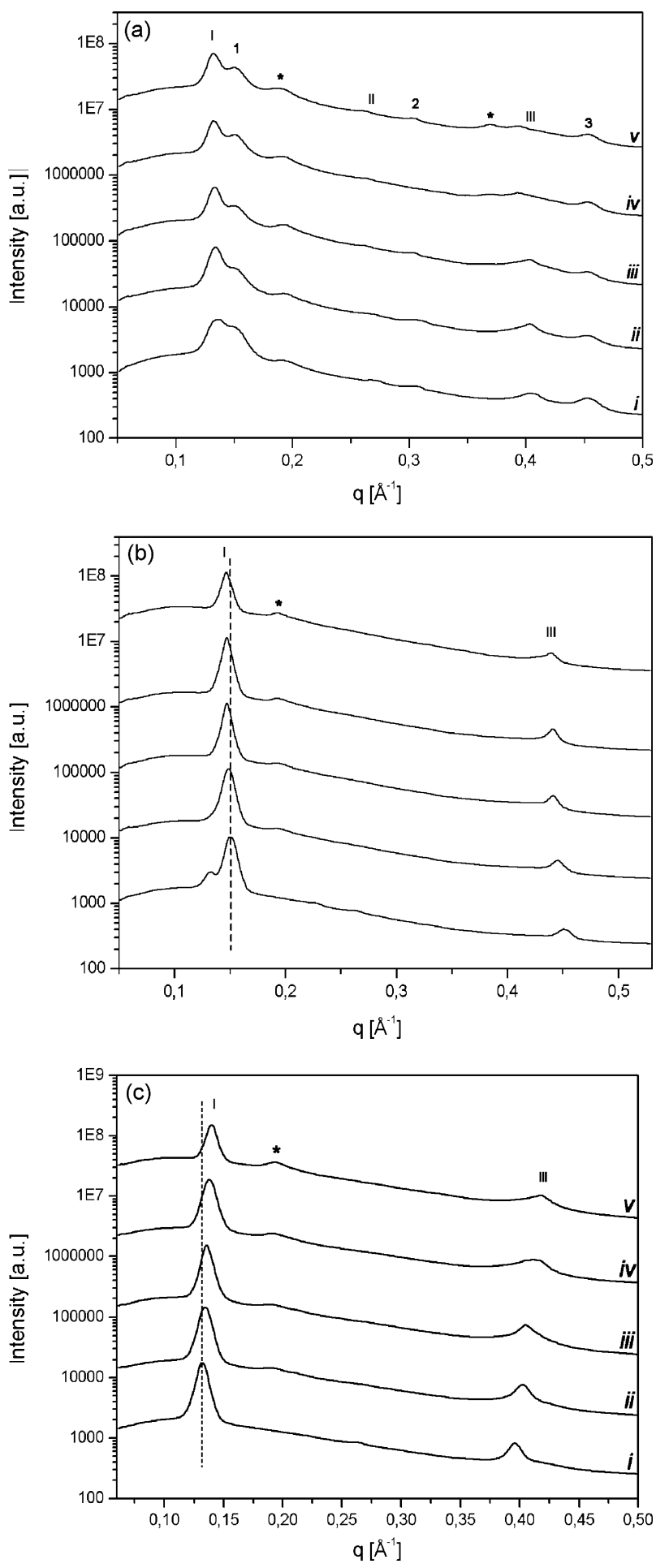

Fig. 3. X-ray diffraction patterns of the SC lipid model system with various cholesterol concentrations; $20 \%$ of lipids in Tris buffer ( $\mathrm{pH} 9)$ in (a) state $\alpha\left(32^{\circ} \mathrm{C}\right.$ before heating), (b) state $\beta\left(85^{\circ} \mathrm{C}\right)$, and (c) state $\delta\left(32^{\circ} \mathrm{C}\right.$ after heating). From bottom to top: (i) $10 \%$ cholesterol, (ii) $15 \%$ cholesterol, (iii) $20 \%$ cholesterol, (iv) $25 \%$ cholesterol, and (v) 30\% cholesterol. The Arabic numerals label the particular reflections of the S-phase; the Roman numerals label the particular reflections of the L-phase; the asterisks label the cholesterol reflections.

IV). No influence of CHOL concentration in the system on the hydrodynamic radius and the polydispersity was found. The ULVs were stable for at least 7 days at $\mathrm{pH} 9$ and laboratory temperature. 
Table 2

Lamellar repeat distances calculated from the X-ray diffraction patterns for the mixtures I-V

\begin{tabular}{|c|c|c|c|}
\hline \multirow[t]{2}{*}{ Sample (cholesterol content) } & \multicolumn{3}{|c|}{ Lamellar repeat distance $(\AA)$ (reflections $\left.\left(\AA^{-1}\right)\right)$} \\
\hline & $32{ }^{\circ} \mathrm{C}$ before heating (state $\alpha$ ) & $85^{\circ} \mathrm{C}$ (state $\beta$ ) & $32^{\circ} \mathrm{C}$ after heating (state $\delta$ ) \\
\hline Mixture I (10\%) & $\begin{array}{l}\mathbf{3 3 . 6} \text { (I: } 0.187) \\
\mathbf{4 1 . 7} \text { (I: } 0.151 \text {; II: } 0.306 \text {; III: } 0.453) \\
\mathbf{4 6 . 7} \AA \text { (I: } 0.135 ; \text { II: } 0.272 ; \text { III: } 0.405 \text { ) }\end{array}$ & 41.8 (I: 0.150; III: 0.451) & $47.6 \AA ̊$ (I: 0.132; III: 0.397) \\
\hline Mixture II (15\%) & $\begin{array}{l}\mathbf{3 3 . 6} \text { (I: } 0.187) \\
\mathbf{4 1 . 8} \text { (I: } 0.150 ; \text { II: } 0.309 ; \text { III: } 0.454 \text { ) } \\
\mathbf{4 7 . 0} \text { (I: } 0.134 \text {; II: } 0.267 ; \text { III: } 0.405 \text { ) }\end{array}$ & $\begin{array}{l}33.6 \text { (I: } 0.187) \\
\mathbf{4 2 . 3} \text { (I: } 0.149 ; \text { III: } 0.446)\end{array}$ & $\begin{array}{l}33.6 \text { (I: } 0.187) \\
\mathbf{4 6 . 9} \text { (I: } 0.134 \text {; III: } 0.403)\end{array}$ \\
\hline Mixture III (20\%) & $\begin{array}{l}\mathbf{3 3 . 6} \text { (I: } 0.187) \\
\mathbf{4 1 . 9} \text { (I: } 0.150 \text {; II: } 0.304 \text {; III: } 0.454 \text { ) } \\
\mathbf{4 7 . 2} \text { (I: } 0.133 \text {; II: } 0.265 ; \text { III: } 0.404 \text { ) }\end{array}$ & $\begin{array}{l}33.6 \text { (I: } 0.187) \\
42.7 \text { (I: } 0.147 ; \text { III: } 0.441)\end{array}$ & $\begin{array}{l}33.6 \text { (I: } 0.187) \\
\mathbf{4 6 . 3} \text { (I: } 0.136 ; \text { III: } 0.408)\end{array}$ \\
\hline Mixture IV (25\%) & $\begin{array}{l}\mathbf{3 3 . 6} \text { (I: } 0.187 \text {; II: } 0.370) \\
\mathbf{4 1 . 9} \text { (I: } 0.150 \text {; III: } 0.455) \\
\mathbf{4 7 . 3} \text { (I: } 0.133 \text {; III: } 0.396)\end{array}$ & $\begin{array}{l}33.6 \text { (I: } 0.187) \\
42.7 \text { (I: } 0.147 ; \text { III: } 0.441)\end{array}$ & $\begin{array}{l}33.6 \text { (I: } 0.187) \\
45.5 \text { (I: } 0.138 ; \text { III: } 0.413)\end{array}$ \\
\hline Mixture V (30\%) & $\begin{array}{l}33.6 \text { (I: } 0.187 ; \text { II: } 0.370) \\
41.9 \text { (I: } 0.150 ; \text { II: } 0.306 ; \text { III: } 0.455) \\
47.5 \AA \text { (I: } 0.132 ; \text { II: } 0.263 ; \text { III: } 0.394)\end{array}$ & $\begin{array}{l}33.6 \text { (I: } 0.187) \\
42.8 \text { (I: } 0.147 ; \text { III: } 0.439)\end{array}$ & $\begin{array}{l}33.6 \text { (I: } 0.187) \\
44.9 \text { (I: } 0.140 \AA \text {; III: } 0.419)\end{array}$ \\
\hline
\end{tabular}

The Roman numerals in brackets describe the particular reflections.

\subsection{Molecular modelling and the average scattering-length density calculation}

The solvent accessible volumes $\left(V_{S A}\right)$ were calculated for Cer[AP], CS, CHOL, and palmitic acid. The $V_{\mathrm{SA}}$-values amount to 990.59 and $666.86 \AA^{3}$ for Cer[AP] and CS, respectively. The volumes of CHOL and palmitic acid are 617.12 and $450.27 \AA^{3}$, respectively, which is comparable with literature data obtained from volumetric measurements [35,36].

The average excess scattering-length density per unit mass $\left(\Delta \rho_{\mathrm{m}}\right)$ of the lipid mixtures in $\mathrm{D}_{2} \mathrm{O}$ was determined from the known chemical composition [37]. The calculated values are listed in Table 3.

\subsection{Small-angle neutron scattering from ULVs}

Fig. 7a shows the neutron scattering curves of the ULVs with various CHOL concentrations. No diffraction peak of a multi- or

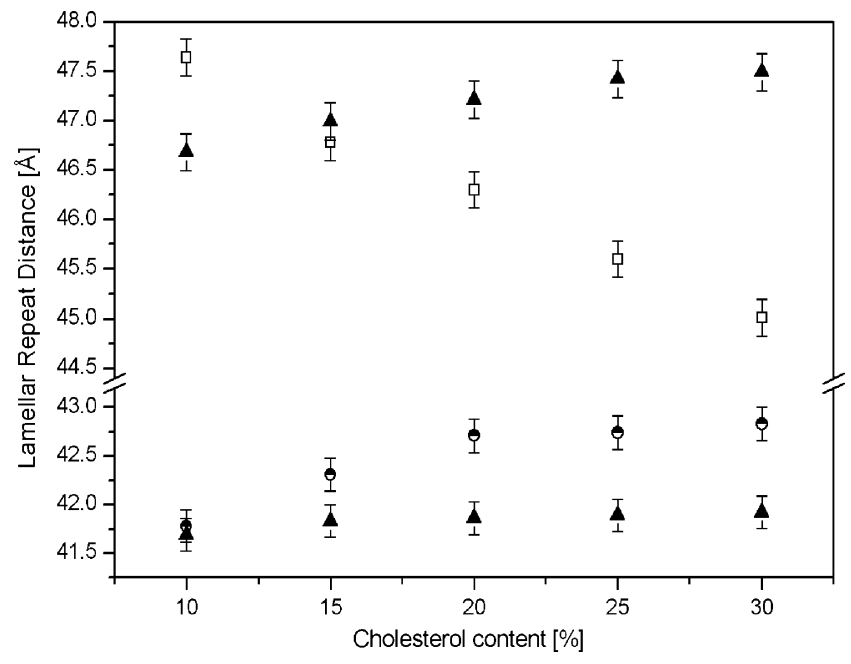

Fig. 4. Lamellar repeat distance of the SC lipid model system with various cholesterol concentrations measured in state $\alpha$ at $32^{\circ} \mathrm{C}$ before heating (filled triangles), state $\beta$ at $85^{\circ} \mathrm{C}$ (partly filled circles), and state $\delta$ at $32^{\circ} \mathrm{C}$ after heating (open squares). The repeat distance was calculated using a Lorentzian function to fit the first order of diffraction.

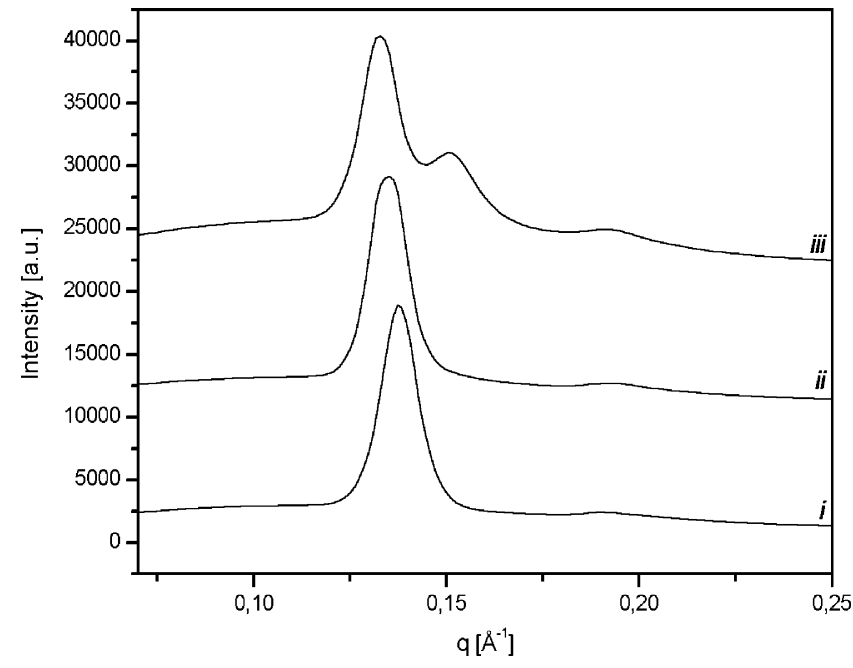

Fig. 5. Recovery of the system with $25 \% \mathrm{CHOL}$ (mixture IV) into the phase-separated state. From bottom to top: system measured (i) $20 \mathrm{~min}$ after the heating, (ii) 1 day after the heating, and (iii) 10 days after the heating (the samples were incubated at $5{ }^{\circ} \mathrm{C}$ during this time).

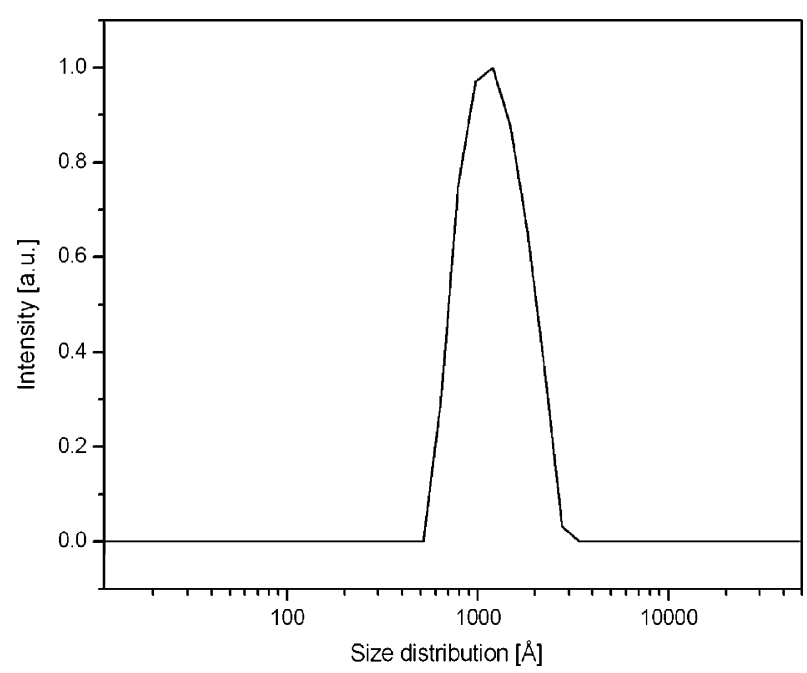

Fig. 6. The size distribution of the mixture IV ( $25 \%$ cholesterol); $1 \%(w / w)$ of lipids in $\mathrm{D}_{2} \mathrm{O}$ buffer extruded through $500 \AA$ filters. 
Table 3

The average excess scattering-length density $\left(\Delta \rho_{\mathrm{m}}\right)$ of the lipid mixtures in $\mathrm{D}_{2} \mathrm{O}$ calculated per $1 \mathrm{~g}$ of lipids

\begin{tabular}{ll}
\hline Mixture & $\Delta \rho_{\mathrm{m}} \times 10^{10}\left(\mathrm{~cm} \mathrm{~g}^{-1}\right)$ \\
\hline I $(10 \%$ CHOL $)$ & -6.198 \\
II $(15 \%$ CHOL $)$ & -6.189 \\
IV $(25 \%$ CHOL $)$ & -6.170 \\
V (30\% CHOL) & -6.159 \\
\hline
\end{tabular}

oligolamellar arrangement or of crystalline CHOL, as in the case of MLVs, is present.

The minimum of the membrane thickness, $q_{\mathrm{d} \text { min }}$, is distinct at about $0.18 \AA^{-1}$ and shifts its position to higher $q$-values with the increasing $\mathrm{CHOL}$ content. The membrane thickness parameters, $d$, calculated from the minimum $q_{\mathrm{d} \text { min }}$ according to the MSFF with an accuracy of $5 \%$ amount to $36.9,36.4,35.3$, and $34.6 \AA$ for mixture I (9\% CHOL after extrusion), II (14\% CHOL), IV (20\% CHOL), and V (27\% CHOL), respectively (Fig. 8a).

The Kratky-Porod plots of the measured scattering curves (Fig. 7b) show a linear range between 0.06 and $0.14 \AA^{-1}$. The slopes of the plots, which correspond to the square of the radius of gyration, decrease with increasing concentration of CHOL in the samples. The membrane thickness parameters, $d_{\mathrm{g}}$, calculated from the $R_{\mathrm{g}}$ values show the same tendency and amount to $38.7 \pm 0.8$, $38.2 \pm 0.8,35.3 \pm 0.7$, and $33.9 \pm 0.7 \AA$ for mixture I, II, IV, and V, respectively (Fig. $8 \mathrm{a}$ ).

The average area per molecule of the membrane surface, $A$, has been calculated from the $I(0)$ values and the average scatteringlength density of the lipid mixtures, as described in Section 2.5. The determined values are 55.2, 57.3, 61.7, and $68.1 \AA^{2}$ for mixture I ( $9 \% \mathrm{CHOL})$, II (14\% CHOL), IV (20\% CHOL), and V (27\% CHOL), respectively. Because the molar volumes were determined by the molecular modelling and not by real measurements, a statistical error can affect the results; however, the tendency of the increasing CHOL concentration to increase the area per molecule of the membrane surface is obvious (Fig. 8b).

The average volume per molecule in the membrane, $V$, has been determined from the average area and the membrane thickness parameter. The values calculated from d according to the MSFF are 1018.4, 1042.9, 1089.0, and $1178.1 \AA^{3}$ for mixtures I (9\% CHOL), II (14\% CHOL), IV (20\% CHOL), and V (27\% CHOL), respectively. For the same samples, the $V$-values obtained from $d_{\mathrm{g}}$ according to the GA are $1068.1,1094.4,1089.0$, and $1154.3 \AA^{3}$, respectively (Fig. $8 \mathrm{c}$ ).

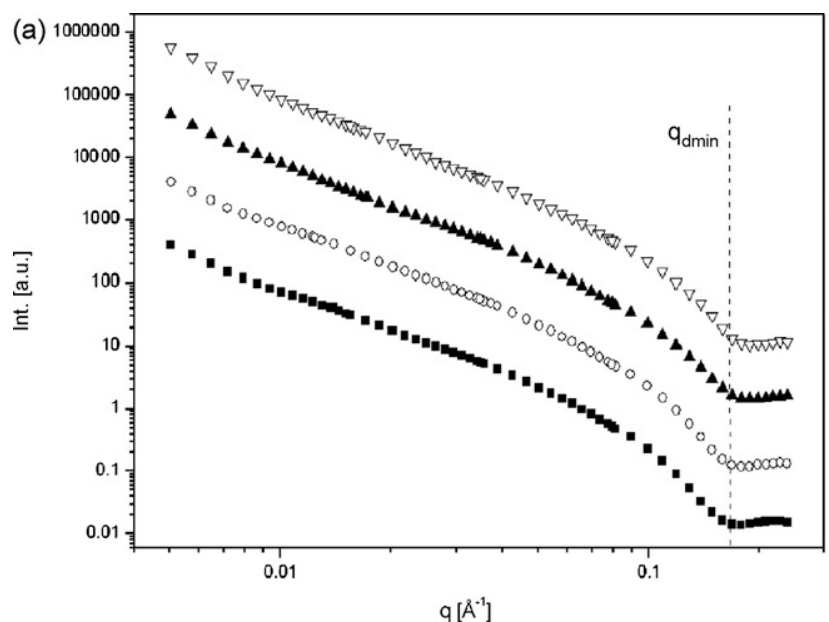

The obtained $V$-values enabled us to calculate the average membrane density, $\rho_{\mathrm{m}}$. Again two data sets have been acquired. For mixtures I, II, IV, and V, the $\rho_{\mathrm{m}}$ values are $0.829,0.799,0.744$, and $0.678 \mathrm{~g} / \mathrm{cm}^{3}$ according to the MSFF, and 0.791, 0.761, 0.744, $0.693 \mathrm{~g} / \mathrm{cm}^{3}$ according to the GA, respectively (Fig. 8d).

The minimum, $q_{\mathrm{R} \text { min }}$, related to the average vesicle radius $[28,38]$, is difficult to observe only for mixture I at $0.008 \AA^{-1}$ which agrees with the vesicle radius of about $400 \AA$ (Fig. 7a). No minimum can be detected in the SANS curves from other samples.

The data obtained from the SANS measurements are summarized in Table 4.

\section{Discussion}

This study introduces SANS on ULVs into the characterization methods of the SC lipid membranes. In order to carry out these measurements successfully, it was important to prepare stable unilamellar vesicles of a SC lipid model system. The composition was chosen according to previous studies showing that to create stable ULVs, high $\mathrm{pH}$ of the environment is required $[18,39]$. Unlike $\mathrm{pH}$ values close to human skin, at $\mathrm{pH} \mathrm{9,} \mathrm{the} \mathrm{charged}$ components (palmitic acid and CS in the present system) are mainly ionized [40]. As a result, vesicles can be extruded without a considerable loss of lipids. Contrary to ULVs prepared at $\mathrm{pH} 6$, vesicles prepared at $\mathrm{pH} 9$ have been found to be stable for 4-6 weeks [18]. A decrease in $\mathrm{pH}$ to 6 activated vesicle fusion and lysis [41]. For comparison, in human SC there is a continual $\mathrm{pH}$ gradient between $\mathrm{pH} 5$ and 7 [42]. Consequently, the degree of ionisation of the fatty acids also varies across the SC [40].

Since the SANS measurements take quite a long time (several days), stable ULVs are needed. For this reason, samples with high $\mathrm{pH}$ values in the environment were used in our experiments. Of course, the $\mathrm{pH}$ difference between the prepared model membranes and the native SC must be taken into consideration while interpreting the results. Nevertheless, this compromise enabled us to apply SANS and to employ the 'model of separated form factors' and the Guinier approximation to characterize the internal membrane structure.

The minimum at $q_{\mathrm{R} \text { min }}$ related to the average vesicle radius, which is normally noticeable in SANS curves of phospholipid vesicles $[28,38]$, can barely be detected at $10 \% \mathrm{CHOL}$. The vesicle radius was determined to be about $400 \AA$. The fact that the minimum is only poorly distinguishable suggests high polydispersity of the

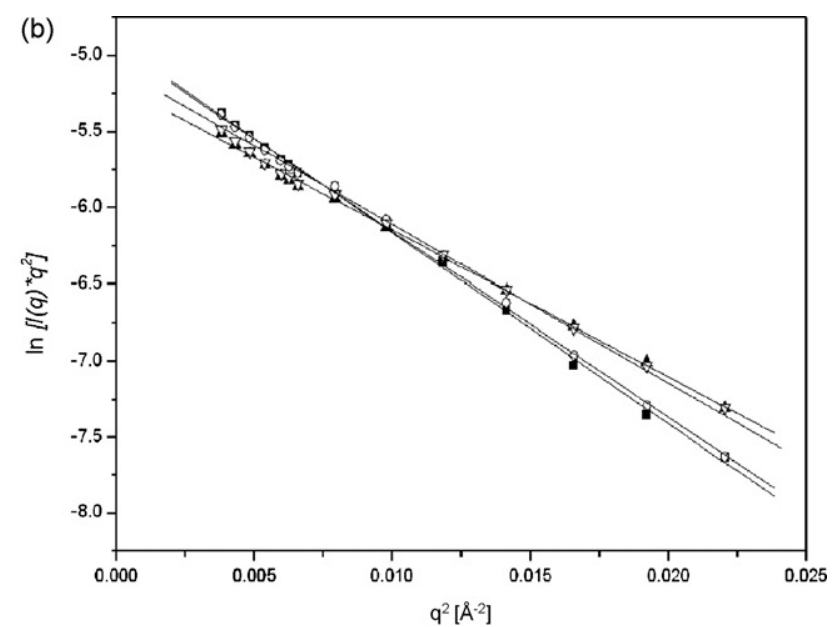

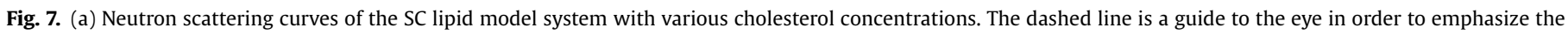

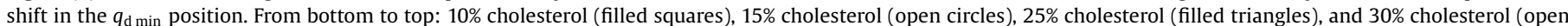
triangles). (b) The corresponding Kratky-Porod plots of the SANS curves (legend: as (a)). 

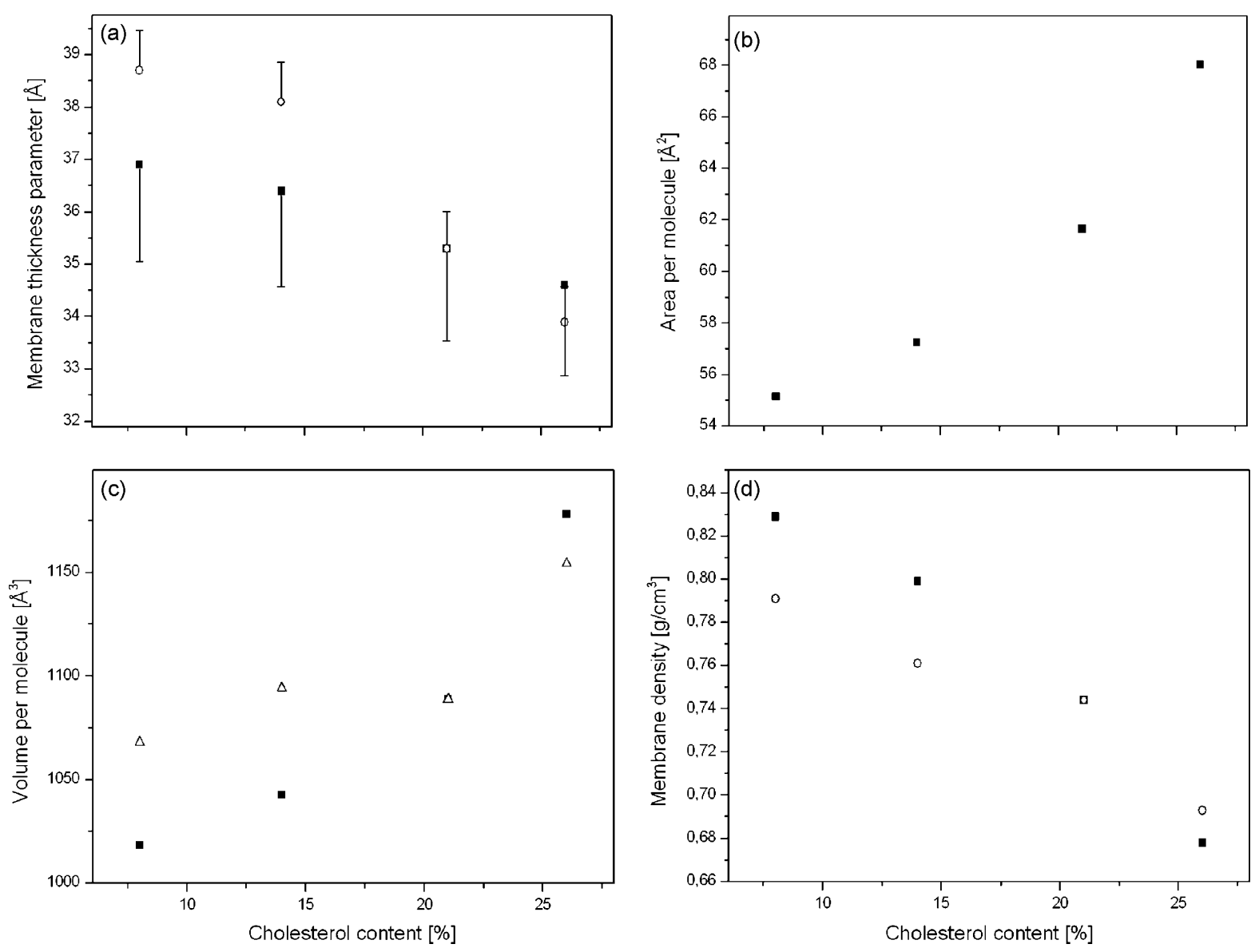

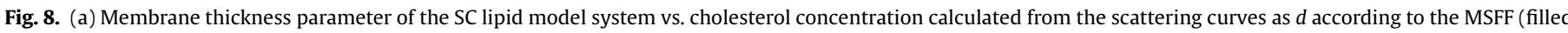

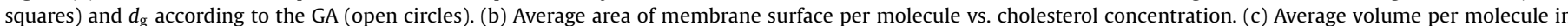

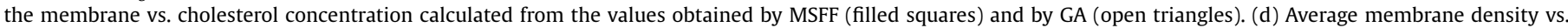
cholesterol concentration calculated from the values obtained by MSFF (filled squares) and by GA (open circles).

sample. The absence of the minimum in the other curves can also indicate, apart from the high polydispersity, that the vesicle radii of the SC lipid ULVs are larger than the detection limit of the SANS measurements (about $550 \AA$ ).

The SAXD measurements have been carried out in three different states of the system at $32^{\circ} \mathrm{C}$ (the temperature of human skin) and $85^{\circ} \mathrm{C}$. According to differential scanning calorimetry (DSC) (Appendix A), the main phase transition of all the samples can be found in the range between 40 and $80^{\circ} \mathrm{C}$ and broadens with the increasing $\mathrm{CHOL}$ concentration in the system. Thus, in the present experiments, the samples were measured below, over, and again below the main phase transition.
Immediately after the preparation, the MLVs show one phase with the lamellar repeat distance of 45.9 and $48.0 \AA$ for 25 and $10 \%$ CHOL, respectively. The components are mixed at the molecular level. This state is not stable for a long-time period and corresponds to the state $\delta$ arising after heating (Fig. 9).

The measurements carried out 10 days after the sample preparation indicate that the lipids separated into three phases (state $\alpha)$.

When the system is then heated over the main phase transition to $85^{\circ} \mathrm{C}$, only one phase is observable again, but the repeat distances, $D$, are different from the $D$-values detected immediately after the preparation (state $\beta$ ).

Table 4

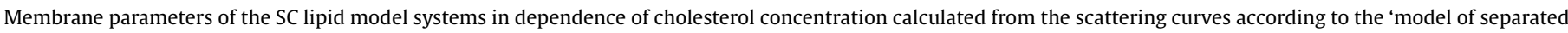
form factors' and the Guinier approximation

\begin{tabular}{|c|c|c|c|c|c|c|c|c|c|}
\hline \multirow[t]{2}{*}{ Evaluation method } & \multicolumn{4}{|c|}{ Model of separated form factors } & \multicolumn{5}{|c|}{ Guinier approximation } \\
\hline & $q_{\mathrm{d} \min }\left(\AA^{-1}\right)$ & $d(\AA)$ & $V\left(\AA^{3}\right)$ & $\rho\left(\mathrm{g} / \mathrm{cm}^{3}\right)$ & $R_{\mathrm{g}}^{2}\left(\AA^{2}\right)$ & $d_{g}(\AA)$ & $A\left(\AA^{2}\right)$ & $V_{g}\left(\AA^{3}\right)$ & $\rho_{\mathrm{g}}\left(\mathrm{g} / \mathrm{cm}^{3}\right)$ \\
\hline \multicolumn{10}{|l|}{ System } \\
\hline $\mathrm{I}(10 / 8.6)^{\mathrm{a}}$ & $0.170 \pm 0.009$ & $36.9 \pm 1.9$ & 1018.4 & 0.829 & $124.7 \pm 2.5$ & $38.7 \pm 0.8$ & 55.2 & 1068.1 & 0.791 \\
\hline II $(15 / 14.2)^{\mathrm{a}}$ & $0.172 \pm 0.009$ & $36.4 \pm 1.8$ & 1042.9 & 0.799 & $121.3 \pm 2.4$ & $38.2 \pm 0.8$ & 57.3 & 1094.4 & 0.761 \\
\hline $\operatorname{IV}(25 / 21.6)^{a}$ & $0.178 \pm 0.009$ & $35.3 \pm 1.8$ & 1089.0 & 0.744 & $103.7 \pm 2.1$ & $35.3 \pm 0.7$ & 61.7 & 1089.0 & 0.744 \\
\hline $\mathrm{V}(30 / 27)^{\mathrm{a}}$ & $0.181 \pm 0.009$ & $34.6 \pm 1.7$ & 1178.1 & 0.678 & $95.5 \pm 1.9$ & $33.9 \pm 0.7$ & 68.1 & 1154.3 & 0.693 \\
\hline
\end{tabular}

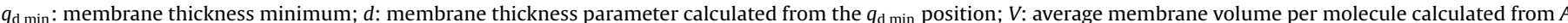

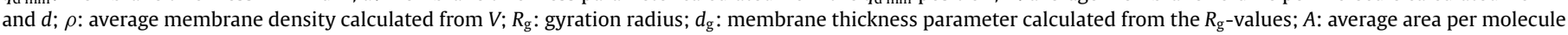
of the membrane surface; $V_{\mathrm{g}}$ : average membrane volume per molecule calculated from $A$ and $d_{\mathrm{g}} ; \rho_{\mathrm{g}}$ : average membrane density calculated from $V_{\mathrm{g}}$.

a $\%$ of cholesterol before and after extrusion. 


\section{preparation \\ $\|$}

state $\delta$

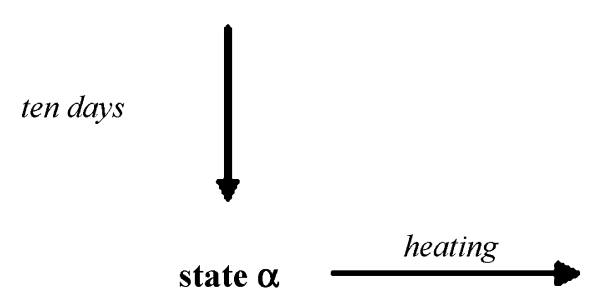

state $\delta$

cooling

\section{state $\beta$}

Fig. 9. A schematic depiction of the phase behaviour of the SC lipid model system.

When the systems are cooled back to $32^{\circ} \mathrm{C}$, they appear in the mixed state $\delta$ again.

In the following, we will focus more carefully on the individual states.

\section{The state $\alpha$}

As mentioned above, in the state $\alpha$, three phases with the repeat distances of approximately 33.6, 42, and $47 \AA$ are present in the system. The phase with a stable repeat distance of $33.6 \AA$ at all CHOL concentrations has been assigned to $\mathrm{CHOL}$ separated into CHOL monohydrate crystals [34]. Such phase separation of CHOL was described previously not only in the phospholipid membranes but also in the native SC and SC lipid model membranes [43,44].

As it was described earlier, ceramides and free fatty acids can phase separate in a system of SC lipids [6]. A similar phase separation probably occurs in the state $\alpha$ of our systems. The repeat distance of the L-phase agrees with $D$-values obtained for a palmitic acid/CHOL system at high $\mathrm{pH}$ as described by Quimet et al. [45]. These authors even showed that with increasing CHOL concentration in the palmitic acid/CHOL system, the lamellar repeat distance slightly increases. As can be seen in Fig. 4, a similar effect can also be observed for the L-phase of the present system suggesting that the L-phase consists mainly of the separated palmitic acid and a part of CHOL.

The effect of the phase separation between fatty acids and ceramides in the SC lipid systems has been interpreted by the mismatch in their chain lengths [46]. In our system, however, the chain length of the ceramide used is comparable with the chain length of palmitic acid.

Considering the L-phase consists of palmitic acid/CHOL, the Sphase must consist mainly of Cer[AP]. However, the repeat distance of 41.9 does not fully correspond with the values obtained for pure Cer[AP] [47]. It can be caused by the fact that in our case a small amount of CHOL is also present in the S-phase.

To confirm the assumption that the L-phase consists mainly of palmitic acid and the S-phase of Cer[AP], further studies are in progress.

\section{The state $\beta$}

Upon heating the phase-separated $\alpha$-state to $85^{\circ} \mathrm{C}$, the lipids mix and only one phase is detected. This phase is affected by the CHOL concentration in the system. With the increasing CHOL con- tent, the repeat distance increases. The increase in the periodicity can be caused by the increase either in the water layer between the membranes or in the membrane thickness. As the water layer between the membranes was described to be extremely thin $(0.8 \AA)$ and only a minimal difference in the water layer was detected between 60 and 99\% hydration for this SC lipid system [16] it is likely that the increasing periodicity is induced mainly by the increasing membrane thickness.

This statement is also in agreement with the generally accepted hypothesis for phospholipid membranes showing that CHOL tends to increase the thickness and decrease the fluidity and permeability of the lipid bilayers in the fluid phase [48-50]. The effect can be explained taking into account that $\mathrm{CHOL}$ increases the conformational order of the hydrocarbon chains of the other lipids, which causes the chain mobility and the membrane fluidity to decrease.

The state $\delta$

After cooling the system back, the components remain mixed and only one phase is observable. The lamellar repeat distance of this phase is close to that described for the same system at lower $\mathrm{pH}$ [16] confirming the reliability of our system using higher $\mathrm{pH}$. Therefore, we consider that the $\delta$-state is the most similar to native SC.

The lamellar repeat distance in the $\delta$-state shows a high sensitivity to the CHOL amount in the membrane. In the concentration range used, the periodicity decreases with increasing CHOL content in the system. This dependence seems to have a linear tendency. The effect of CHOL on the membrane in this state can be interpreted in several ways or, of course, a combination of them:

(1) CHOL increases the tilt angle with respect to the base plane of the membrane. Besides the decreasing $D$-values with the increasing CHOL amount in the membrane, the decreasing membrane thickness and the increasing average membrane area detected by SANS should be concomitant effects when the tilt angle increases [51]. (2) CHOL decreases the order of the hydrocarbon chains of the participating lipids. Consequently, the increased fluidity of the chains can be the reason for the decreasing $D$-values. The finding that the area per molecule of the membrane surface increases with the increasing CHOL concentration in the system can also support this view. The increasing area/molecule of the membrane surface indicates that the intermolecular interactions between the particular lipids ( $\mathrm{H}$ bonds in the head group region) weaken, which is again connected with the increase in the membrane fluidity. It was published for phospholipids that CHOL evokes a similar increase in area/molecule in the gel phase, which causes an increase in diffusion coefficient through the membranes [48]. The increase in the average volume per molecule, which we have detected by SANS, suggests a possible decrease in the chain order of the lipids as well. Clear proof that CHOL induces the decrease in the lipid chain order and thereby increases the membrane fluidity, however, is the decreasing membrane density evoked by CHOL (Fig. 8d).

The difference between the lamellar repeat distances and the membrane thickness parameter is not caused by the water layer between the membranes as one could suppose, but by the fact that the membrane thickness parameter mostly describes only that region of the membrane where no $\mathrm{D}_{2} \mathrm{O}$ penetration exists; it means the hydrophobic part of the membrane-the hydrocarbon core region.

According to Small [52], it is possible to calculate the theoretical hydrocarbon chain length in the all-trans (most rigid) conformation. Calculating the theoretical length of the longest chain in the present mixture (the stearoyl-chain in Cer[AP]), one obtains a value of $22.9 \AA$ per chain. Adding up these two values, the thickness of the hydrocarbon chain part of the membrane should be $45.8 \AA$. 
There appears to be a large difference between this theoretical value and the $d$ or $d_{\mathrm{g}}$ values obtained by SANS. This difference can be attributed to the tilting of the hydrocarbon chains to the base plane of the membrane at an angle $\theta$. Moreover, a partial interdigitation of the stearoyl chains in the membrane centre is very probable, because various chain lengths are present in the membrane.

In summary, for phospholipids, it was described that the addition of CHOL restricts the motion of lipids and condenses the membranes above the main phase transition temperature and, on the contrary, fluidises the membranes below the temperature $[53,54]$. The aforesaid results show a similar effect of CHOL on the SC model lipid membrane consisting mainly of ceramides with no phospholipids. In the case of phospholipid membranes, this effect has been explained by CHOL dual act of inducing high conformational order in the lipid chains and, simultaneously, breaking the lateral packing order of the lipid molecules. As a result, CHOL facilitates formation of the liquid ordered phase in phospholipid bilayers $[50,55]$. Possibly, CHOL can influence the SC lipid membranes in a similar way. This assumption, however, requires further investigations.

In conclusion, the result of this study that CHOL decreases the order and increases the fluidity of the SC lipid membranes at $32{ }^{\circ} \mathrm{C}$, which is the temperature of the human skin, is of special importance in RXLI. Therefore, the role of CHOL in the SC lipid membranes must be taken into consideration in the studies of RXLI pathophysiology.

\section{Conclusions}

The influence of CHOL on the structure of a model SC lipid membrane was studied. Besides X-ray diffraction, SANS on ULVs was newly applied. This technique enabled us to determine the membrane thickness parameter, area of the membrane surface, membrane volume per molecule, and membrane density. The present data support the hypothesis that $\mathrm{CHOL}$ decreases the order of the well-ordered hydrocarbon chains in the state below the main phase transition and otherwise increases the chain order in the state above the main phase transition in a SC lipid membrane.

\section{Acknowledgements}

The work was supported by the Grant Agency of the Czech Republic (Project GACR 202/07/P391), by the Ministry of Education of the Czech Republic (Grant MSM 0021620822) and by the Federal State of Saxony-Anhalt, Germany (Project 3482A/1102L). We thank Dr. Kateřina Vávrová for fruitful discussions and Dr. Christoph Wagner for his assistance with the X-ray measurements. Thanks are also extended to Cosmoferm for the gift of Cer[AP] as well as to DESY and GKSS Research Centre for the travel and accommodation reimbursements.

\section{Appendix A. Supplementary data}

Supplementary data associated with this article can be found, in the online version, at doi:10.1016/j.colsurfa.2008.06.032.

\section{References}

[1] M. Ponec, A. Weerheim, P. Lankhorst, P. Wertz, New acylceramide in native and reconstructed epidermis, J. Invest. Dermatol. 120 (2003) 581-588.

[2] D. Webster, J.T. France, L.J. Shapiro, R. Weiss, X-linked ichthyosis due to steroidsulphatase deficiency, Lancet 1 (8055) (1978) 70-72.

[3] M.L. Williams, P.M. Elias, Stratum corneum lipids in disorders of cornification, J. Clin. Invest. 68 (1981) 1404-1410.

[4] E. Zettersen, M.-Q. Man, J. Sato, M. Denda, A. Farell, R. Ghadially, M.L. Williams, K.R. Feingold, P.M. Elias, Recessive X-linked ichthyosis: role of cholesterol- sulphate accumulation in the barrier abnormality, J. Invest. Dermatol. 111 (1998) 784-790.

[5] M.L. Williams, K.R. Feingold, G. Grubauer, P.M. Elias, Ichtyosis induced by cholesterol-lowering drugs. Implications for epidermal cholesterol homeostasis, Arch. Dermatol. Res. 123 (1987) 1535-1538.

[6] M. Lafleur, Phase behaviour of model stratum corneum lipid mixtures: an infrared spectroscopy investigation, Can. J. Chem. 76 (1998) 1501-1511.

[7] M. Wegener, R. Neubert, W. Rettig, S. Wartewig, Structure of stratum corneum lipids characterized by FT-Raman spectroscopy and DSC. III. Mixtures of ceramides and cholesterol, Chem. Phys. Lipids 88 (1997) 73-82.

[8] H.-C. Chen, R. Mendelsohn, M.E. Rerek, D.J. Moore, Effect of cholesterol on miscibility and phase behavior in binary mixtures with synthetic ceramide 2 and octadecanoic acid. Infrared studies, Biochim. Biophys. Acta 1512 (2001) 345-356.

[9] E. Sparr, L. Eriksson, J.A. Bouwstra, K. Ekelund, AFM study of lipid monolayers. III. Phase behavior of ceramides, cholesterol and fatty acids, Langmuir 17 (2001) 164-172.

[10] J. Pencer, S. Krueger, C.P. Adams, J. Katsaras, Method of separated form factors for polydisperse vesicles, J. Appl. Cryst. 39 (2006) 293-303.

[11] N. Kučerka, M.A. Kiselev, P. Balgavý, Determination of bilayer thickness and lipid surface area in unilamellar dimyristoylphosphatidylcholine vesicles from small-angle neutron scattering curves: a comparison of evaluation methods, Eur. Biophys. J. 33 (2004) 328-334.

[12] G. Ch. Charalambopoulou, T.A. Steriotis, A.Ch. Mitropoulos, K.L. Stefanopoulos, N.K. Kanellopoulos, Investigation of water sorption on porcine stratum corneum by very small angle neutron scattering, J. Invest. Dermatol. 110 (1998) 988-990.

[13] G. Ch. Charalambopoulou, P. Karamertzanis, E.S. Kikkinides, A.K. Stubos, N.K. Kanellopoulos, A. Th. Papaioannou, A Study on structural and diffusion properties of porcine stratum corneum based on very small angle neutron scattering data, Pharm. Res. 17 (2000) 1085-1091.

[14] G. Ch. Charalambopoulou, Th. A. Steriotis, Th. Hauss, K.L. Stefanopoulos, A.K. Stubos, A neutron-diffraction study of the effect of hydration on stratum corneum structure, Appl. Phys. A 74 (Suppl.) (2002) S1245S1247.

[15] G. Ch. Charalambopoulou, Th. A. Steriotis, Th. Hauss, A.K. Stubos, N.K. Kanellopoulos, Structural alterations of fully hydrated human stratum corneum, Physica B 350 (2004) e603-e606.

[16] M.A. Kiselev, N.Y. Ryabova, A.M. Balagurov, S. Dante, T. Hauss, J. Zbytovská, S. Wartewig, R. Neubert, New insights into the structure and hydration of a stratum corneum lipid model membrane by neutron diffraction, Eur. Biophys. J. 34 (2005) 1030-1040.

[17] G. Ch. Charalambopoulou, Th. A. Steriotis, K.L. Stefanopoulos, A. Ch. Mitropoulos, N.K. Kanellopoulos, U. Keiderling, Investigation of lipid organization on stratum corneum by water absorption in conjunction with neutron scattering, Physica B 276-278 (2000) 530-531.

[18] R.M. Hatfield, L.W.M. Fung, Molecular properties of a stratum corneum model lipid system: large unilamellar vesicles, Biophys. J. 68 (1995) 196-207.

[19] W. Abraham, D.T. Downing, Preparation of model membranes for skin permeability studies using stratum corneum lipids, J. Invest. Dermatol. 93 (1989) 809-813.

[20] R.R.C. New, Liposomes-A Practical Approach, IRL Press at Oxford University Press, Oxford, New York, Tokyo, 1990

[21] H. Farwanah, R. Neubert, S. Zellmer, K. K. Raith, Improved procedure for the separation of major stratum corneum lipids by means of automated multiple development thin-layer chromatography, J. Chromatogr. B 780 (2002) 443-450.

[22] http://www.esrf.eu/computing/scientific/FIT2D/.

[23] M.A. Kiselev, P. Lesieur, A.M. Kisselev, D. Lombardo, V.L. Aksenov, Model of separated form factors for unilamellar vesicles, Appl. Phys. A 74 (2002) S1654-S1656.

[24] W. Knoll, J. Haas, H.B. Stuhrmann, H.-H. Fuldner, H. Vogel, E. Sackmann, Smallangle neutron scattering of aqueous dispersions of lipids and lipid mixtures. A contrast variation study, J. Appl. Cryst. 14 (1981) 191-202.

[25] L.A. Feigin, D.I. Svergun, Structure Analysis by Small-angle X-ray and Neutron Scattering, Plenum Publishing Corporation, New York, 1987.

[26] P. Balgavý, M. Dubničková, N. Kučerka, M.A. Kiselev, S.P. Yaradaikin, D. Uhríková, Bilayer thickness and lipid interface area in unilamellar extruded 1,2diacylphosphatidylcholine liposomes: a small-angle neutron scattering study, Biochim. Biophys. Acta 1512 (2001) 40-52.

[27] M.A. Kiselev, D. Lombardo, A.M. Kisselev, P. Lesieur, V.L. Aksenov, Structure factor of dimyristoylphosphatidylcholine unilamellar vesicles: small-angle X-ray scattering study, Poverhnost 11 (2003) 20-24 (written in Russian).

[28] M.A. Kiselev, J. Zbytovská, D. Matveev, S. Wartewig, I.V. Gapienko, J. Perez, P. Lesieur, A. Hoell, R. Neubert, Influence of trehalose on the structure of unilamellar DMPC vesicles, Colloids Surf. A 256 (2005) 1-7.

[29] V.M. Garamus, J.S. Pedersen, H. Kawasaki, H. Maeda, Scattering from polymer like micelles of TDAO in salt/water solutions at semidilute concentrations, Langmuir 16 (2000) 6431-6437.

[30] J.F. Nagle, S. Tristam-Nagle, Structure of lipid bilayers, Biochim. Biophys. Acta 1469 (2000) 159-195.

[31] K. Vávrová, J. Zbytovská, K. Palát, T. Holas, J. Klimentová, A. Hrabálek, P. Doležal, Ceramide analogue 14S24 ((S)-2-tetracosanoylamino-3-hydroxypropionic acid tetradecyl, Pharm. Sci. 21 (2004) 581-587.

[32] N. Bodor, Z. Gabanyi, C. Wong, A new method for the estimation of partition coefficients, J. Am. Chem. Soc. 111 (1989) 3783-3786. 
[33] A. Gavezzotti, The calculation of molecular volumes and the use of volume analysis in the investigation of structured media and of solid state organic reactivity, J. Am. Chem. Soc. 105 (1983) 5220-5225.

[34] B.M. Craven, Crystal structure of cholesterol monohydrate, Nature 260 (1976) 727-729.

[35] E. Ayranci, G. Akgul, Apparent molar volumes and viscosities of lauric, palmitic and stearic acids in 2-butanol at 20,30,40, and $60^{\circ} \mathrm{C}$, J. Chem. Eng. Data 48 (2003) 56-60.

[36] P. Góralski, M. Wasiak, Influence of van der Waals interactions on volumetric properties of cholesterol in solvents of linear structure, J. Chem. Thermodyn. 35 (2003) 1623-1634.

[37] Y. Chevalier, Y. Zemb, The structure of micelles and microemulsions, Rep. Prog. Phys. 53 (1990) 279-371.

[38] J.Zbytovská, M.A. Kiselev, S.S. Funari, V. Garamus, S. Wartewig, R. Neubert, Influence of phytosphingosine-type ceramides on the structure of DMPC membrane Chem. Phys. Lipids 138 (2005) 69-80.

[39] D.T. Downing, W. Abraham, B. Wegner, K.W. Willman, J.E. Marshall, Partition of sodium dodecyl sulphate into stratum corneum lipid liposomes, Arch. Dermatol. Res. 285 (1993) 151-157.

[40] R. Lieckfeldt, J. Vallalaín, J.-C. Gómez-Fernández, G. Lee, Apparent $\mathrm{p} K_{\mathrm{a}}$ of the fatty acids within ordered mixtures of model human stratum corneum lipids, Pharm. Res. 12 (1995) 1614-1617.

[41] R.M. Hatfield, L.W.M. Fung, A new model system for lipid interaction in stratum corneum vesicles: effects of lipid composition, calcium, and $\mathrm{pH}$, Biochemistry 38 (1999) 784-791.

[42] H. Öhman, A. Vahlquist, In vivo studies concerning a pH gradient in human stratum corneum and upper epidermis, Acta Derm. Venerol. 74 (1994)375-379.

[43] J. Huang, G.W. Feigenson, A microscopic interaction model of maximum solubility of cholesterol in lipid bilayers, Biophys. J. 76 (1999) 2142-2157.

[44] J.A. Bouwstra, G.S. Gooris, W. Bras, D.T. Downing, Lipid organization in pig stratum corneum, J. Lipid Res. 36 (1995) 685-695.
[45] J. Quimet, S. Croft, Ch. Paré, J. Katsaras, M. Lafleur, Modulation of the polymorphism of the palmitic acid/cholesterol system by the $\mathrm{pH}$, Langmuir 19 (2003) 1089-1097.

[46] J.A. Bouwstra, G.S. Gooris, K. Cheng, A. Weerheim, W. Bras, M. Ponec, Phase behaviour of isolated skin lipids, J. Lipid Res. 37 (1996) 999-1011.

[47] S. Raudenkolb, S. Wartewig, R.H.H. Neubert, Polymorphism of ceramide 6: a vibrational spectroscopic and X-ray powder diffraction investigation of the diastereomers of $\mathrm{N}$-( $\alpha$-hydroxyoctadecanoyl)-phytosphingosine, Chem. Phys. Lipids 133 (2005) 89-102.

[48] O.G. Mouritsen, M.J. Zuckermann, What's so special about cholesterol? Lipids 39 (2004) 1101-1113.

[49] M.R. Vist, J.H. Davis, Phase equilibria of cholesterol/dipalmitoylphosphatidylcholine mixtures: $2 \mathrm{H}$ nuclear magnetic resonance and differential scanning calorimetry, Biochemistry 29 (1990) 451-464.

[50] L. Miao, M. Nielsen, J. Thewalt, J.H. Ipsen, M. Bloom, M.J. Zuckermann, O.G. Mouritsen, From lanosterol to cholesterol: structural evolution and differential effects on lipid bilayers, Biophys. J. 82 (2002) 1429-1444.

[51] G. Pabst, S. Danner, S. Karmakar, G. Deutsch, V.A. Raghunathan, On the propensity of phosphatidylglycerols to form interdigitated phases, Biophys. J. 93 (2007) 513-525.

[52] D.M. Small, The Physical Chemistry of Lipids, Plenum Press, New York, London, 1986

[53] D. Needham, T.J. McIntosh, E. Evans, Thermomechanical and transition properties of dimyristoylphosphatidylcholine/cholesterol bilayers, Biochemistry 27 (1988) 4668-4673.

[54] R.A. Demel, B. De Kruyff, The function of sterols in membranes, Biochim. Biophys. Acta 457 (1976) 109-132.

[55] J.H. Ipsen, G. Karlsröm, O.G. Mouritsen, H. Wennersröm, M.J. Zuckermann, Phase equilibria in the phosphatidycholine-cholesterol system, Biochim. Biophys. Acta 905 (1987) 162-172. 